\title{
Ironing out the angles in p53
}

The presence of mutated p53 in approximately $50 \%$ of all human tumours has led to an intense interest in the structure and function of the protein. In vivo $\mathrm{p} 53$ functions as a tetramer and it is this ability to oligomerize that is responsible for the dominant negative phenotypes associated with some mutant forms of p53. Earlier structures of the tetramerization domain, determined by nuclear magnetic resonance (NMR) spectroscopy ${ }^{1,2}$, have significant differ-

\section{IMAGE UNAVAILABLE FOR COPYRIGHT REASONS}

Fig. 1 Differing quaternary structures. Comparison of the NMR and $X$-ray structures of the 533 oligomerization domain: $a$, NMR structure of Clore et $a l .{ }^{1} ; b$, NMR structure of Lee et $a I^{2} ; c$, refined NMR structure of Clore et $a l^{3}{ }^{3}$; and $d$, X-ray structure of Jeffery et $a .^{4}$. The A (red) and C (yellow) subunits (residues 325-355) form one dimer and the $B$ (green) and $D$ (blue) subunits form the other dimer of the p53 tetramer. The $A B$ (and by symmetry $C D$ ) interhelical angles are; $a, 114^{\circ} ; b, 57^{\circ} ; c, 81^{\circ}$; and $d$, $80^{\circ}$ (calculated with the program Define_Structure ${ }^{6}$; images generated with the program VISP'; figure kindly provided by G.M.Clore). ences in their quaternary conformations; three new structures, one in this issue of Nature Structural Biology $y^{3}$ and two in Science $e^{4.5}$, may now put this conflict to rest.

Genetic and biochemical evidence implicates p53 in the regulation of the cell cycle; in particular, p53 is involved in monitoring the integrity of genomic DNA before replication. For this reason the protein has been dubbed the 'guardian of the genome.' The $\mathrm{p} 53$ protein is a sequence-specific DNA-binding protein and transcription factor; downstream targets include the gene for p21, whose product inhibits cyclin-dependent kinase-4, thereby blocking cell division.

There are four domains in p53: an aminoterminal transcriptional activation domain; a central 'core' domain that mediates sequencespecific DNA-binding; an oligomerization domain; and a basic carboxy-terminal, regulatory domain. The structure of the minimal tetramerization domain ${ }^{1-5}$ consists of a dimer of dimers, each dimer being constructed from a two-stranded antiparallel $\beta$-sheet and two antiparallel $\alpha$-helices (Fig 1). The interface between the two dimers is formed by the two pairs of $\alpha$-helices which come together in a four-helix bundle motif: the $\beta$-sheets (which are crucial in the formation of the dimer) lie on the outside of the bundle.

Although they share a common topology, the original two NMR structures ${ }^{1,2}$ are, in terms of their quaternary structure, noticeably different from one another (Fig. $1 a, b$ ). The most obvious difference is in the way the dimers pack against one another, that is, in the angle between the long axes of the pairs of helices from each dimer that contribute to the four-helix bundle in the tetramer. There are also subtle differences in the degree of bending of the $\alpha$-helices. The question must then be whether these differences in conformation are real: does the tetramerization domain exist in a number of different forms (which may have implications 
for function) or are the differences artifacts of the structure determination?

The high resolution $(1.7 \AA)$ X-ray crystal structure of the oligomerization domain has now been solved ${ }^{4}$. This led Clore, Gronenborn and colleagues ${ }^{5}$ to reassess the data used to generate their original NMR structure': they now find a number of discrepancies. In particular, a couple of the nuclear Overhauser enhancements (NOEs), used to pin down the position of the two dimers relative to each other, were missed. In addition, three very weak NOEs at the interface of the two dimers were found to be incorrect, actually arising from the $4 \mathrm{D}{ }^{13} \mathrm{C} /$ ${ }^{13} \mathrm{C}$-separated NOE spectrum. NOE data provide information on short range $(<5 \AA)$ through-space interactions between protons. The assignment of NOEs between residues that are distant from one another in the primary sequence but close in the tertiary (or quaternary) structure plays a particularly important part in determining structural models that can be fitted to the collection of NMR restraints.

The absence of a few key NOEs as well as the presence of the three incorrect NOEs, has been compounded by both the inherent limitations of the NMR experiments, as applied to a symmetric oligomer, and the structure of the tetramerization domain. The quality of an NMR structure is for the most part gauged by the average number of restraints per residue. It is of course the actual number of restraints for any particular residue that will determine the accuracy of that part of the structure. Relatively few NOEs are observed between the dimers in the original structure from Clore et al. ${ }^{1}$; a mere $24(\times 4)$ between the interdimer $A$ and $B$ subunits compared with $190(\times 4)$ NOEs between the intradimer $A$ and $C$ subunits. Although this need not necessarily be a problem if the NOEs nail down the critical elements of the structure, errors in such 'data-poor' regions can have a dramatic effect on the final structure model.

A further stumbling-block is provided by the nature of the intersubunit interface in the tetramer. The two dimers pack together in an orthogonal manner; the helices from one dimer cross over at an angle to those from the other dimer (Fig. 1). As a consequence there are more interactions (and
NOEs) between the helices at the crossing point and relatively few at the ends of the helices. And yet it is these latter interactions that are crucial for pinning down the orientation of the dimers relative to each other. Unluckily for Clore, Gronenborn and colleagues ${ }^{1}$ three vital NOEs out towards the end of the helices were missed in the original spectra which, along with the three spurious NOEs across the dimer-dimer interface, resulting in the two dimers being oriented at an angle of $114^{\circ}$ (defined by the interhelical angle between the $\mathrm{A}(\mathrm{red})$ and $\mathrm{B}$ (green) helices), compared to $\sim 80^{\circ}$ for the new structures $^{3.4}$ (Fig.1). The Lee et al. structure ${ }^{2}$ is also different from the new structures ${ }^{3,4}$, for example, the $\mathrm{AB}$ interhelical angle is somewhat smaller, $\sim 60^{\circ}$. Arrowsmith and colleagues suggest that this is primarily due to curvature of the helices away from the dimer interface at the $\mathrm{C}$-terminus in their structure rather than a difference in rigid body rotation of one dimer relative to the other.

Further analysis of the NMR data by Clore, Gronenborn and colleagues ${ }^{3}$ has resulted in more than 4,400 structural restraints, with a three-anda-half-fold increase in the number of intersubunit NOEs at the dimer interface, yielding a structure that is essentially identical in tertiary and quaternary conformation to the crystal structure (Fig. 1b,c).The consensus between the latest structures suggests that they are representative of the conformation of the domain. And such knowledge is important. Any attempts to engineer p 53 or to design drugs against mutant forms of the protein will require details of its precise conformation if effective therapies are to be developed. What the structure of the oligomerization domain will be in the intact molecule and its complex with DNA remains to be seen. Let us hope the wait is not too long.

1. Clore, G.M. et al. Science 265, 386-391 (1994).

2. Lee et al. Nature struct. Biol. 1, 877890 (1994).

3. Clore, G.M et al. Nature struct. Biol. 2, 321-333 (1995)

4. Jeffrey P.D., Gorina, S. \& Pavletich, N.P.

Science 267, 1498-1502 (1995).

5. Clore, G.M. et al. Science 267, 1515-

1516 (1995).

6. Richards, F.M. \& Kundrot, C.E. Proteins 3, 71-84 (1988).

7. de Castro, E. \& Edelstein, S. VISP 1.0 User's Guide (University of Geneva, Switzerland, 1992). 